Translation from Russian

of the article published in

ZNS POMI, 285, 39-52 (2002)

\title{
Coherent states \\ for the Legendre oscillator ${ }^{1}$
}

\author{
V.V.Borzov, ${ }^{*}$ E.V.Damaskinsky ${ }^{* *}$ \\ * St.-Petersburg University of Telecommunications \\ E-mail: vadim@VB6384.spb.edu \\ ${ }^{* *}$ St.-Petersburg University of the Defence Engineering Constructions \\ E-mail: evd@pdmi.ras.ru
}

\begin{abstract}
A new oscillator-like system called by the Legendre oscillator is introduced in this note. The two families of coherent states (coherent states as eigenvectors of the annihilation operator and the Klauder - Gazeau temporally stable coherent states) are defined and investigated for this oscillator.
\end{abstract}

\section{Introduction}

In the present note we construct coherent states for an oscillator-like system called by the Legendre oscillator.Note that the Legendre polynomials play the same role for this system as the Hermite polynomials for standard boson oscillator.

\footnotetext{
${ }^{1}$ This research is supported in part by RFFI grant no. 00-01-00500.
} 
It is known that in the case of harmonic oscillator three standard definitions of coherent state (as eigenvector of an annihilation operator; as state generated by a shift operator from the vacuum state and as state minimizing the uncertainty relation) are equivalent.This means that they generate the same set of states. However, in the general case this is not so. Below we shall construct coherent state connected with the Legendre polynomials. This construction results from the analysis [1] of the linkage between orthogonal polynomials and generalized oscillator algebras [3, 2. Namely, we shall define for the Legendre oscillator analogues of Barut - Girardello coherent states [5] and Gazeau - Klauder coherent states [6]. The more detailed exposition is demanded for definition of coherent states of the Perelomov-type [7, 8, 9]. So it will be postponed to the other publication.

\section{The Legendre oscillator.}

For the reader convenience we remind some information about the Legendre polynomials. The Legendre polynomials $P_{(x)}$ are the solutions of the differential equations

$$
\left(1-x^{2}\right) y^{\prime \prime}-2 x y^{\prime}+n(n+1) y=0, \quad y(x)=P_{n}(x)
$$

which satisfy the orthogonality condition

$$
\int_{-1}^{1} P_{n}(x) P_{m}(x) \mathrm{d} x=\frac{2}{2 n+1} \delta_{n m} .
$$

They also are solutions of the following recurrent relations

$$
(2 n+1) x P_{n}(x)=(n+1) P_{n+1}(x)+n P_{n-1}(x) ; \quad P_{0}(x)=1 ; \quad\left(P_{-1}(x)=0\right), \quad n \in \mathbb{N}_{0} .
$$

The Legendre polynomials are defined by the relation $\left(n \in \mathbb{N}_{0}\right)$

$$
\begin{aligned}
P_{n}(x)={ }_{2} \mathrm{~F}_{1}\left(\begin{array}{c|c}
-n, n+1 \\
1
\end{array} \mid \frac{1-x}{2}\right) & =\sum_{m=0}^{\llbracket \frac{n}{2} \rrbracket} \frac{(-1)^{m}}{2^{n}}\left(\begin{array}{c}
n \\
m
\end{array}\right)\left(\begin{array}{c}
2 n-2 m \\
n
\end{array}\right) x^{n-2 m}= \\
& =\sum_{m=0}^{\llbracket \frac{n}{2} \rrbracket} \frac{(-1)^{m}(2 n-2 m) !}{2^{n} m !(n-m) !(n-2 m) !} x^{n-2 m},
\end{aligned}
$$

where a symbol $\llbracket n \rrbracket$ denotes the integer part of a number $n$. Below we shall use the following generating function for this polynomials

$$
\sum_{n=0}^{\infty} \frac{(\gamma)_{n}}{n !} P_{n}(x) z^{n}=(1-x z)_{2}^{-\gamma} \mathrm{F}_{1}\left(\begin{array}{c}
\frac{1}{2} \gamma, \frac{1}{2}(1+\gamma) \\
1
\end{array} \mid \frac{\left(x^{2}-1\right) z^{2}}{(1-x z)^{2}}\right) ; \quad|x| \leq 1,|z| \leq 1
$$


Simultaneously with the Legendre polynomials we consider the Legendre functions

$$
\psi_{n}(x)=\sqrt{2 n+1} P_{n}(x), \quad n \in \mathbb{N}_{0},
$$

which form a orthonormal basis $\left\{|n\rangle \equiv \psi_{n}(x)\right\}_{n=0}^{\infty}$ in the Hilbert space

$$
\mathcal{H}:=L^{2}\left([-1,1], \frac{1}{2} \mathrm{~d} x\right) .
$$

These functions fulfill the recurrent relations

$$
x \psi_{n}(x)=b_{n-1} \psi_{n-1}(x)+b_{n} \psi_{n+1}(x), \quad \psi_{-1}(x)=0, \quad \psi_{0}(x)=1,
$$

with coefficients

$$
b_{n}=\sqrt{\frac{(n+1)^{2}}{(2 n+1)(2 n+3)}}, \quad n \geq 0 .
$$

In the given research the Legendre polynomials $P_{n}(x)$ and the Legendre functions $\psi_{n}(x)$ play the same role as the Hermite polynomials and the Hermite functions play in the standard quantum mechanics.

In the Hilbert space $\mathcal{H}$ we define the generalized position operator $X$ connected with the Legendre polynomials $P_{n}(x)$ as an operator of multiplication by argument:

$$
X|n\rangle=x|n\rangle .
$$

Taking into account a relation (8), we have

$$
X \psi_{n}(x)=b_{n} \psi_{n+1}(x)+b_{n-1} \psi_{n-1}(x),
$$

where the coefficients $b_{n}$ are defined by the relation (19). Because $\sum_{k=0}^{\infty} \frac{1}{b_{k}}=\infty$, the operator $X$ is a selfajoint operator in the space $\mathcal{H}$ (see [10, 11, 2]) .

Let us define a generalized momentum operator $P$ by the way described in [1]. The operator $P$ acts on the basis elements in $\mathcal{H}$ by the following formula

$$
P|n\rangle=i\left(b_{n}|n+1\rangle-b_{n-1}|n-1\rangle\right) .
$$

Calculating usual commutator of operators $X$ and $P$ on the basis elements, we obtain

$$
[X, P]|n\rangle=2 i\left(b_{n}{ }^{2}-b_{n-1}{ }^{2}\right)|n\rangle=\frac{2 i}{(2 n-1)(2 n+1)(2 n+3)}|n\rangle .
$$

Now we define the creation and annihilation operators by the standard relations

$$
a^{(+)}=\frac{1}{\sqrt{2}}(X-i P), \quad a^{(-)}=\frac{1}{\sqrt{2}}(X+i P) .
$$


On the basis elements in $\mathcal{H}$ these operators act by the rule

$$
a^{(+)}|n\rangle=\sqrt{2} b_{n}|n+1\rangle, \quad a^{(-)}|n\rangle=\sqrt{2} b_{n-1}|n-1\rangle .
$$

They satisfy the commutation relations

$$
\left[a^{(-)}, a^{(+)}\right]=\frac{1}{i}[X, P] .
$$

Now we introduce the state numbering operator $N$ and Hamiltonian $H$, by the following formulae

$$
N|n\rangle=n|n\rangle, \quad H=X^{2}+P^{2}=a^{(+)} a^{(-)}+a^{(-)} a^{(+)} .
$$

The eigenvalues of the operator $H$ are equal to

$$
\lambda_{0}=2 b_{0}{ }^{2}, \quad \lambda_{n}=2\left({b_{n-1}}^{2}+b_{n}{ }^{2}\right) .
$$

It is natural call the introduced system by the Legendre oscillator.

\section{Barut - Girardello coherent states for the Legendre oscillator}

In this section we define the coherent states for the Legendre oscillator in the space $\mathcal{H}$ as eigenvectors of the annihilation operator $a^{(-)}$

$$
a^{(-)}|z\rangle=z|z\rangle .
$$

It is known that

$$
|z\rangle=\mathcal{N}^{-1} \sum_{n=0}^{\infty} \frac{z^{n}}{\left(\sqrt{2} b_{n-1}\right) !}|n\rangle .
$$

The normalizing factor is equal to

$$
\mathcal{N}^{2}=\langle z \mid z\rangle=\sum_{n=0}^{\infty} \frac{|z|^{2 n}}{\left(2 b_{n-1}{ }^{2}\right) !} \equiv \exp _{\left[2 b_{n-1}{ }^{2}\right]}\left(|z|^{2}\right)
$$

Because of ${ }^{2}$

$$
\left(2 b_{n-1}^{2}\right) !=\frac{2^{n}(n !)^{2}}{(2 n-1) ! !(2 n+1) ! !}=\frac{(n !)(1)_{n}}{2^{n}\left(\frac{1}{2}\right)_{n}\left(\frac{3}{2}\right)_{n}},
$$

\footnotetext{
${ }^{2}$ Here $(a)_{n}$ is the Pochhammer symbol defined by the relation

$$
(a)_{0}=1,(a)_{n}=a(a+1) \cdots(a+n-1)=\frac{\Gamma(a+n)}{\Gamma(a)}, \quad n=1,2, \ldots
$$
}


the radius of convergence of a series (21) equals to $\frac{1}{\sqrt{2}}$ and

$$
\mathcal{N}^{2}=\sum_{n=0}^{\infty} \frac{\left(\frac{1}{2}\right)_{n}\left(\frac{3}{2}\right)_{n}}{n !(1)_{n}}\left(2|z|^{2}\right)^{n}={ }_{2} \mathrm{~F}_{1}\left(\left.\begin{array}{c}
\frac{1}{2}, \frac{3}{2} \\
1
\end{array}|2| z\right|^{2}\right)
$$

Substituting (23) in (20) and using a relation $|n\rangle=\psi_{n}(x)=\sqrt{2 n+1} P_{n}(x)$, we obtain

$$
\begin{aligned}
|z\rangle & =\left[{ }_{2} \mathrm{~F}_{1}\left(\left.\begin{array}{c}
\frac{1}{2}, \frac{3}{2} \\
1
\end{array}|2| z\right|^{2}\right)\right]^{-\frac{1}{2}} \sum_{n=0}^{\infty} \frac{(2 n-1) ! !(2 n+1)}{n !} P_{n}(x) z^{n}= \\
& =\left[{ }_{2} \mathrm{~F}_{1}\left(\left.\begin{array}{c}
\frac{1}{2}, \frac{3}{2} \\
1
\end{array}|2| z\right|^{2}\right)\right]^{-\frac{1}{2}} \sum_{n=0}^{\infty} \frac{\left(\frac{3}{2}\right)_{n}(\sqrt{2} z)^{n}}{n !} P_{n}(x) .
\end{aligned}
$$

From(5) as $\gamma=\frac{3}{2}$ and $z \rightarrow 2 z$ we obtain

$$
|z\rangle=\left[{ }_{2} \mathrm{~F}_{1}\left(\left.\begin{array}{c}
\frac{1}{2}, \frac{3}{2} \\
1
\end{array}|2| z\right|^{2}\right)\right]_{2}^{-\frac{1}{2}} \mathrm{~F}_{1}\left({ }_{1}^{\frac{3}{4}, \frac{5}{4}} \mid \frac{\left(x^{2}-1\right) 2 z^{2}}{(1-\sqrt{2} x z)^{2}}\right)(1-\sqrt{2} x z)^{-\frac{3}{2}}
$$

Our following task is to construct a measure

$$
\mathrm{d} \mu\left(|z|^{2}\right)=W\left(|z|^{2}\right) \mathrm{d}^{2} z, \quad \text { such that } \iint_{\mathbb{C}} W\left(|z|^{2}\right)|z\rangle\langle z| \mathrm{d}^{2} z=1
$$

where $\mathrm{d}^{2} z=\mathrm{d}(\operatorname{Re} z) \mathrm{d}(\operatorname{Im} z)$. It is known (see, for example, [12]), that this problem is reduced to a solution of the following the Hausdorf moment problem

$$
\sum_{n=0}^{\infty} \frac{\pi}{\left(2 b_{n-1}^{2}\right) !}\left[\int_{0}^{\frac{1}{2}} t^{n} W(t) \mathrm{d} t\right]|n\rangle\langle n|=1, \quad\left(t=|z|^{2}\right)
$$

or

$$
\int_{0}^{\frac{1}{2}} t^{n} W(t) \mathrm{d} t=\frac{1}{\pi}\left(2 b_{n-1}^{2}\right) !
$$

Substituting (22) in (27), we have (for $\tau=2 t$ )

$$
\frac{1}{2} \int_{0}^{1} \tau^{n} W\left(\frac{1}{2} \tau\right) \mathrm{d} \tau=\frac{1}{\pi} \frac{(n !)^{2}}{\left(\frac{1}{2}\right)_{n}\left(\frac{3}{2}\right)_{n}} .
$$

So, it is necessary to solve a following Hausdorf moment problem

$$
\frac{1}{2} \int_{0}^{1} \tau^{n} W\left(\frac{1}{2} \tau\right) \mathrm{d} \tau=\frac{1}{\pi} \frac{(\Gamma(n+1))^{2} \Gamma\left(\frac{1}{2}\right) \Gamma\left(\frac{3}{2}\right)}{\Gamma\left(n+\frac{1}{2}\right) \Gamma\left(n+\frac{3}{2}\right)}
$$


or, taking into the account $\Gamma\left(\frac{1}{2}\right) \Gamma\left(\frac{3}{2}\right)=\frac{1}{2} \pi$,

$$
\int_{0}^{1} \tau^{n} W\left(\frac{1}{2} \tau\right) \mathrm{d} \tau=\frac{(\Gamma(n+1))^{2}}{\Gamma\left(n+\frac{1}{2}\right) \Gamma\left(n+\frac{3}{2}\right)}, \quad n \geq 0
$$

Making in an integral (see (7.127) in [13])

$$
J=\int_{-1}^{1}(1+x)^{\sigma} P_{\nu}(x) \mathrm{d} x=\frac{2^{1+\sigma}(\Gamma(1+\sigma))^{2}}{\Gamma(2+\sigma+\nu) \Gamma(1+\sigma-\nu)}, \quad \operatorname{Re} \sigma>-1,
$$

the replacement $x=2 \tau-1$, we receive

$$
J=\int_{0}^{1} 2^{\sigma+1} \tau^{\sigma} P_{\nu}(2 \tau-1) \mathrm{d} \tau .
$$

This allows us to rewrite (30) in the form

$$
\int_{0}^{1} \tau^{\sigma} P_{\nu}(2 \tau-1) \mathrm{d} \tau=\frac{(\Gamma(\sigma+1))^{2}}{\Gamma(\sigma+\nu+2) \Gamma(1+\sigma-\nu)}
$$

Choosing $\sigma=n \operatorname{AND} \nu=\frac{1}{2}$, we obtains

$$
\int_{0}^{1} \tau^{n}\left(P_{\frac{1}{2}}(2 \tau-1)-\tau^{\frac{1}{2}}\right) \mathrm{d} \tau+\int_{0}^{1} \tau^{n+\frac{1}{2}} \mathrm{~d} \tau=\frac{(\Gamma(n+1))^{2}}{\left(n+\frac{3}{2}\right) \Gamma\left(n+\frac{1}{2}\right) \Gamma\left(n+\frac{3}{2}\right)} .
$$

Let's denote

$$
\tau^{-\frac{1}{2}} P_{\frac{1}{2}}(2 \tau-1)-1=\int_{\tau}^{1} q(t) \mathrm{d} t
$$

After differentiating, we find function $q(\tau)$ for $0<\tau<1$

$$
q(\tau)=-\left(\tau^{-\frac{1}{2}} P_{\frac{1}{2}}(2 \tau-1)\right)^{\prime} .
$$

For an investigation of a singularity, arising at $\tau \rightarrow 0^{+}$, we shall consider an integral

$$
\int_{0}^{1} \tau^{n+\frac{1}{2}}\left(\int_{\tau}^{1} q(t) \mathrm{d} t\right) \mathrm{d} \tau
$$

Integrating by parts, we obtain

$$
\int_{0}^{1} \tau^{n+\frac{1}{2}}\left(\int_{\tau}^{1} q(t) \mathrm{d} t\right) \mathrm{d} \tau=\left.\frac{\tau^{n+\frac{3}{2}}}{n+\frac{3}{2}} \int_{\tau}^{1} q(t) \mathrm{d} t\right|_{0} ^{1}+\int_{0}^{1} q(\tau) \frac{\tau^{n+\frac{3}{2}}}{n+\frac{3}{2}} \mathrm{~d} \tau
$$

Let's remark, that

$$
\int_{\tau}^{1} q(t) \mathrm{d} t \underset{\tau \rightarrow 1}{\longrightarrow} 0 \quad \text { and } \quad \tau^{3 / 2} \int_{\tau}^{1} q(t) \mathrm{d} t \underset{\tau \rightarrow 0}{\longrightarrow} 0
$$


from which it follows, that the term outside the integral in (35) is equal to zero. From (32) and (33) it follows, that the integral in the left hand side of the relation (35) is equal to

$$
\int_{0}^{1} \tau^{n+\frac{1}{2}}\left(\int_{\tau}^{1} q(t) \mathrm{d} t\right) \mathrm{d} \tau+\frac{1}{n+\frac{3}{2}}=\frac{(\Gamma(n+1))^{2}}{\left(n+\frac{3}{2}\right) \Gamma\left(n+\frac{1}{2}\right) \Gamma\left(n+\frac{3}{2}\right)},
$$

so that for $n \geq 0$ we have

$$
\int_{0}^{1} q(\tau) \tau^{n+\frac{3}{2}} \mathrm{~d} \tau+1=\frac{(\Gamma(n+1))^{2}}{\Gamma\left(n+\frac{1}{2}\right) \Gamma\left(n+\frac{3}{2}\right)} .
$$

Thus the moment problem is solved by the distribution

$$
W\left(\frac{1}{2} \tau\right)=\tau^{\frac{3}{2}} q(\tau)+2 \delta(\tau-1)=-\tau^{\frac{3}{2}}\left(\tau^{-\frac{1}{2}} P_{\frac{1}{2}}(2 \tau-1)\right)^{\prime}+2 \delta(\tau-1)
$$

or

$$
W(t)=-(2 t)^{\frac{3}{2}}\left((2 t)^{-\frac{1}{2}} P_{\frac{1}{2}}(4 t-1)\right)^{\prime}+2 \delta(2 t-1), \quad 0<t \leq \frac{1}{2} .
$$

Using the formula $(8.832(1))$ from [13], we obtain

$$
W(t)=\frac{(16 t-5) P_{\frac{1}{2}}(4 t-1)-3 P_{\frac{3}{2}}(4 t-1)}{2(2 t-1)}+2 \delta(2 t-1), \quad 0<t \leq \frac{1}{2} .
$$

Finally, for $0<|z| \leq \frac{1}{\sqrt{2}}$ we have

$$
\mathrm{d} \mu\left(|z|^{2}\right)=\left[\frac{\left(16|z|^{2}-5\right) P_{\frac{1}{2}}\left(4|z|^{2}-1\right)-3 P_{\frac{3}{2}}\left(4|z|^{2}-1\right)}{2\left(2|z|^{2}-1\right)}+2 \delta\left(2|z|^{2}-1\right)\right] \mathrm{d}(\operatorname{Re} z) \mathrm{d}(\operatorname{Im} z) .
$$

We calculate overlap of two coherent states

$$
\begin{aligned}
\left\langle z_{1} \mid z_{2}\right\rangle & =\left[{ }_{2} \mathrm{~F}_{1}\left(\left.\begin{array}{c}
\frac{1}{2}, \frac{3}{2} \\
1
\end{array}|2| z_{1}\right|^{2}\right){ }_{2} \mathrm{~F}_{1}\left(\left.\begin{array}{c}
\frac{1}{2}, \frac{3}{2} \\
1
\end{array}|2| z_{2}\right|^{2}\right)\right]^{-\frac{1}{2}} \sum_{n=0}^{\infty} \frac{\bar{z}_{1}^{n} z_{2}{ }^{n}}{\left(2 b_{n-1}{ }^{2}\right) !} \stackrel{\text { 22 }}{=} \\
& =\left[{ }_{2} \mathrm{~F}_{1}\left(\left.\begin{array}{c}
\frac{1}{2}, \frac{3}{2} \\
1
\end{array}|2| z_{1}\right|^{2}\right){ }_{2} \mathrm{~F}_{1}\left(\left.\begin{array}{c}
\frac{1}{2}, \frac{3}{2} \\
1
\end{array}|2| z_{2}\right|^{2}\right)\right]^{-\frac{1}{2}} \sum_{n=0}^{\infty} \frac{\left(2 \bar{z}_{1} z_{2}\right)^{n}\left(\frac{1}{2}\right)_{n}\left(\frac{3}{2}\right)_{n}}{(n !)(1)_{n}} \stackrel{(23)}{=} \\
& =\left[{ }_{2} \mathrm{~F}_{1}\left(\left.\begin{array}{c}
\frac{1}{2}, \frac{3}{2} \\
1
\end{array}|2| z_{1}\right|^{2}\right){ }_{2} \mathrm{~F}_{1}\left(\left.\begin{array}{c}
\frac{1}{2}, \frac{3}{2} \\
1
\end{array}|2| z_{2}\right|^{2}\right)\right]^{-\frac{1}{2}}{ }_{2} \mathrm{~F}_{1}\left(\frac{1}{2}, \frac{3}{2} \mid 2 \bar{z}_{1} z_{2}\right) .
\end{aligned}
$$

To arbitrary normalized state $|f\rangle=\sum_{n=0}^{\infty} f_{n}\left|\psi_{n}\right\rangle \in \mathcal{H}\left(\sum_{n=0}^{\infty} f_{n}{ }^{2}=1\right)$ we can put in correspondence a function analytical on $\mathbb{C}_{1 / \sqrt{2}}$, by the rule

$$
f(z)=\mathcal{N}(z)\langle z \mid f\rangle=\sum_{n=0}^{\infty} \sqrt{\left(\frac{1}{2}\right)_{n}\left(\frac{3}{2}\right)_{n}} \frac{f_{n}}{n !}(2 z)^{n}
$$


so that after expansion on coherent states we have

$$
|f\rangle=\int_{\mathbb{C}_{1 / \sqrt{2}}}\langle z \mid f\rangle|z\rangle \mathrm{d} \mu\left(|z|^{2}\right)=\int_{\mathbb{C}_{1 / \sqrt{2}}}\left[{ }_{2} \mathrm{~F}_{1}\left(\left.\begin{array}{c}
\frac{1}{2}, \frac{3}{2} \\
1
\end{array}|2| z\right|^{2}\right)\right]^{-\frac{1}{2}} f(z)|z\rangle \mathrm{d} \mu\left(|z|^{2}\right)
$$

and

$$
\langle f \mid f\rangle=\int_{\mathbb{C}_{1 / \sqrt{2}}}\left[{ }_{2} \mathrm{~F}_{1}\left(\left.\begin{array}{c}
\frac{1}{2}, \frac{3}{2} \\
1
\end{array}|2| z\right|^{2}\right)\right]^{-1}|f(z)|^{2} \mathrm{~d} \mu\left(|z|^{2}\right)<\infty .
$$

\section{Klauder - Gazeau coherent states for the Legendre oscillator.}

The Klauder - Gazeau temporary stable coherent states [6] is convenient to apply in a case when the Hamiltonian $H$ is nonlinear and its terms are not generators of group of a symmetry. These states can be defined by the relation

$$
|J, \gamma\rangle:=\mathcal{N}(J)^{-1} \sum_{n=0}^{\infty} \frac{J^{\frac{n}{2}}}{\sqrt{\rho_{n}}} \mathrm{e}^{-i \gamma \lambda_{n}}\left|\psi_{n}\right\rangle
$$

where $\rho_{n}=\lambda_{1} \lambda_{2} \cdot \ldots \cdot \lambda_{n}, \quad n \geq 1 ; \quad \rho_{0}=1$, and the normalizing coefficient is equal

$$
\mathcal{N}(J)^{2}=\sum_{n=0}^{\infty} \frac{J^{n}}{\rho_{n}}
$$

Parameters $J$ and $\gamma$ takes the values $J \geq 0, \gamma \in \mathbb{R}$. Note that these parameters are generalization of the module and argument (extended up to an infinite covering of a segment [0;2 $]$ ) of a standard parameter $z=|z| \mathrm{e}^{i \gamma}$ of coherent states. One can to consider these parameters as analogue of the classical action - angle variables.

In considered case the Hamiltonian $H$ is a positive selfajoint operator in a Hilbert space $\mathcal{H}$

with a simple discrete (ordered by decreasing) spectrum $\left\{\lambda_{n}=2 b_{n-1}^{2}\right\}_{n=1}^{\infty}$, where $b_{n}$ is taken from (9), so that we can write

$$
\begin{aligned}
& \rho_{0}=1, \rho_{n}=\left(2 b_{n-1}{ }^{2}\right) !=\frac{(n !)(1)_{n}}{2^{n}\left(\frac{1}{2}\right)_{n}\left(\frac{3}{2}\right)_{n}}, \quad n \geq 1, \\
& \mathcal{N}(J)^{2}=\sum_{n=0}^{\infty} J^{n} \frac{2^{n}\left(\frac{1}{2}\right)_{n}\left(\frac{3}{2}\right)_{n}}{(n !)(1)_{n}}={ }_{2} \mathrm{~F}_{1}\left(\begin{array}{c}
\frac{1}{2}, \frac{3}{2} \\
1
\end{array} \mid 2 J\right) .
\end{aligned}
$$


The radius of convergence of a series in (47) is equal to $R=\lim _{n \rightarrow \infty} \sqrt[n]{\rho_{n}}=\frac{1}{2}$. Taking into account (46), (47), we obtain

$$
|J, \gamma\rangle=\frac{1}{\sqrt{{ }_{2} \mathrm{~F}_{1}\left(\begin{array}{c}
\frac{1}{2}, \frac{3}{2} \\
1
\end{array} \mid 2 J\right)}} \sum_{n=0}^{\infty} \frac{\sqrt{2 n+1}}{n !} J^{n / 2} 2^{n / 2} \sqrt{\left(\frac{1}{2}\right)_{n}\left(\frac{3}{2}\right)_{n}} \mathrm{e}^{-i \sqrt{2} b_{n-1 \gamma}} P_{n}(x),
$$

or, because $\left(\frac{3}{2}\right)_{n}=(2 n+1)\left(\frac{1}{2}\right)_{n}$,

$$
|J, \gamma\rangle=\frac{1}{\sqrt{{ }_{2} \mathrm{~F}_{1}\left(\frac{1}{2}, \frac{3}{2} \mid 2 J\right)}} \sum_{n=0}^{\infty} \frac{2 n+1}{n !}(2 J)^{n / 2}\left(\frac{1}{2}\right)_{n} \mathrm{e}^{-i \sqrt{2} b_{n-1} \gamma} P_{n}(x) .
$$

To proof the validity of the resolution of identity we consider the relation

$$
\int|J, \gamma\rangle\langle J, \gamma| \mathrm{d} \mu(J, \gamma)=\lim _{T \rightarrow \infty} \frac{1}{2 T} \int_{T}^{T} \mathrm{~d} \gamma\left[\int_{0}^{\infty} k(J)|J, \gamma\rangle\langle J, \gamma| \mathrm{d} J\right],
$$

where

$$
k(J):=\left\{\begin{array}{cc}
\mathcal{N}(J)^{2} \rho(J) & 0 \leq J \leq \frac{1}{2} \\
0 & J>\frac{1}{2}
\end{array} .\right.
$$

Calculating an integral over $\gamma$ we obtain

$$
\int|J, \gamma\rangle\left\langle J, \gamma\left|\mathrm{d} \mu(J, \gamma)=\sum_{n=0}^{\infty} \frac{1}{\rho_{n}} \int_{0}^{\frac{1}{2}}\right| n\right\rangle\langle n| \mathrm{d} J .
$$

Thus, the resolution of identity

$$
\int|J, \gamma\rangle\langle J, \gamma| \mathrm{d} \mu(J, \gamma)=\mathbb{I}
$$

is fulfilled, if the weight function $\rho(J)$ gives a solution of a moment problem

$$
\int_{0}^{\frac{1}{2}} J^{n} \rho(J) \mathrm{d} J=\rho_{n}=\frac{n !(1)_{n}}{2^{n}\left(\frac{1}{2}\right)_{n}\left(\frac{3}{2}\right)_{n}}, \quad n \geq 0 .
$$

In view of the relations (27) and (38) a solution of this problem is given by the relation $(0<$ $\left.J<\frac{1}{2}, \gamma \in \mathbb{R}\right)$

$$
\rho(J)=\frac{\pi}{4(2 J-1)}\left[(16 J-5) P_{\frac{1}{2}}(4 J-1)-3 P_{\frac{3}{2}}(4 J-1)\right]+\pi \delta(2 J-1) .
$$


The temporal stability is obvious, as

$$
\mathrm{e}^{-i H t}|J, \gamma\rangle=\frac{1}{\mathcal{N}} \sum_{n=0}^{\infty} \frac{J^{n / 2}}{\sqrt{\rho_{n}}} \mathrm{e}^{-i \gamma \lambda_{n}} \mathrm{e}^{-i t \lambda_{n}}\left|\psi_{n}\right\rangle=|J, \gamma+t\rangle .
$$

We have also

$$
\langle J, \gamma|H| J, \gamma\rangle=\mathcal{N}^{-2}(J) \sum_{n=0}^{\infty} \frac{2 b_{n-1}^{2}}{\left(2 b_{n-1}^{2}\right) !} J^{n}=J .
$$

The overlap of two states is given by a relation

$$
\left\langle J^{\prime}, \gamma^{\prime} \mid J, \gamma\right\rangle=\frac{1}{N(J) N\left(J^{\prime}\right)} \sum_{n=0}^{\infty} \frac{\left(J J^{\prime}\right)^{n / 2}}{\rho_{n}} \exp \left[-i \lambda_{n}\left(\gamma-\gamma^{\prime}\right)\right],
$$

which, in the concrete case $\gamma=\gamma^{\prime}$, is easily summarized

$$
\left\langle J^{\prime}, \gamma \mid J, \gamma\right\rangle=\frac{{ }_{2} \mathrm{~F}_{1}\left(\begin{array}{c}
\frac{1}{2}, \frac{3}{2} \\
1
\end{array} \mid 2 \sqrt{J J^{\prime}}\right)}{\sqrt{{ }_{2} \mathrm{~F}_{1}\left(\begin{array}{c}
\frac{1}{2}, \frac{3}{2} \\
1
\end{array} \mid 2 J\right){ }_{2} \mathrm{~F}_{1}\left(\begin{array}{c}
\frac{1}{2}, \frac{3}{2} \\
1
\end{array} \mid 2 J^{\prime}\right)}} .
$$

Taking into account a possible physical applications, we calculate, for example, some quantities having an immediate physical sense. So, for an average number of excitation we have

$$
\begin{aligned}
& \langle n\rangle=\sum_{n=0}^{\infty} n \frac{J^{n}}{\mathcal{N}^{2}(J) \rho_{n}}=\mathcal{N}^{-2} \sum_{n=1}^{\infty} \frac{(2 J)^{n} n\left(\frac{1}{2}\right)_{n}\left(\frac{3}{2}\right)_{n}}{n !(1)_{n}}= \\
& =\frac{2 J}{N^{2}} \sum_{n=0}^{\infty} \frac{(2 J)^{n} \frac{3}{4}\left(\frac{3}{2}\right)_{n}\left(\frac{5}{2}\right)_{n}}{n !(2)_{n}}=\frac{3 J}{2} \frac{{ }_{2} \mathrm{~F}_{1}\left(\begin{array}{c}
\frac{3}{2}, \frac{5}{2} \\
2
\end{array} \mid 2 J\right)}{{ }_{2} \mathrm{~F}_{1}\left(\begin{array}{c}
\frac{1}{2}, \frac{3}{2} \\
1
\end{array} \mid 2 J\right)} .
\end{aligned}
$$

Using the formulas $(7.3 .2(50))$ and $(7.3 .2(217))$ from [14], we obtain the expression for $\langle n\rangle$ in terms of an elliptic integrals

$$
\langle n\rangle=\frac{1}{2} \frac{J}{1-2 J} \frac{2 \mathbf{K}(\sqrt{2 J})-(1+2 J) \mathbf{D}(\sqrt{2 J})}{\mathbf{E}(\sqrt{2 J})},
$$

where

$$
\begin{aligned}
& \mathbf{E}(k)=\int_{0}^{\frac{\pi}{2}} \sqrt{1-k^{2} \sin ^{2} t} \mathrm{~d} t \quad-\text { a full elliptic integral of a 2-nd kind, } \\
& \mathbf{D}(k)=\int_{0}^{\frac{\pi}{2}} \frac{\sin ^{2} t}{\sqrt{1-k^{2} \sin ^{2} t}} \mathrm{~d} t=D\left(\frac{\pi}{2}, k\right) \quad \text { - a full elliptic integral, } \\
& \mathbf{K}(k)=\int_{0} \frac{\pi}{2} \mathrm{~d} \frac{1}{\sqrt{1-k^{2} \sin ^{2} t}} t=F\left(\frac{\pi}{2}, k\right)-\text { a full elliptic integral of a 1-st kind. }
\end{aligned}
$$


The similar evaluations give us that

$$
\begin{aligned}
\left\langle n^{2}\right\rangle & =\sum_{n=0}^{\infty} n^{2} \frac{J^{n}}{N(J)^{2} \rho_{n}}=N^{-2} \sum_{n=1}^{\infty} \frac{(2 J)^{n} \frac{1}{2}\left(\frac{3}{2}\right)^{n-1} \frac{3}{2}\left(\frac{5}{2}\right)^{n-1}}{(n-1) !(1)_{n-1}}= \\
& =\frac{3}{2} \frac{J}{N^{2}} \sum_{n=0}^{\infty} \frac{(2 J)^{n}}{n !} \frac{\left(\frac{3}{2}\right)_{n}\left(\frac{5}{2}\right)_{n}}{(1)_{n}}=\frac{3}{2} J \frac{{ }_{2} \mathrm{~F}_{1}\left(\begin{array}{c}
\frac{3}{2}, \frac{5}{2} \\
1
\end{array} \mid 2 J\right)}{{ }_{2} \mathrm{~F}_{1}\left(\frac{1}{2}, \frac{3}{2} \mid 2 J\right)} .
\end{aligned}
$$

In terms of elliptic integrals we can rewrite (65)

$$
\left\langle n^{2}\right\rangle=\frac{1}{2} \frac{J}{(1-2 J)^{2}} \frac{(3+10 J) \mathbf{K}(\sqrt{2 J})-2 J(7+2 J) \mathbf{D}(\sqrt{2 J})}{\mathbf{E}(\sqrt{2 J})} .
$$

The computation of the variance gives the following relation

$$
\Delta n=\frac{\sqrt{\frac{3}{2} J}}{{ }_{2} \mathrm{~F}_{1}\left(\begin{array}{c}
\frac{1}{2}, \frac{3}{2} \\
1
\end{array} \mid 2 J\right)}\left[{ }_{2} \mathrm{~F}_{1}\left(\frac{3}{2}, \frac{5}{1} \mid 2 J\right){ }_{2} \mathrm{~F}_{1}\left(\frac{1}{2}, \frac{3}{2} \mid 2 J\right)-\frac{3}{2} J\left({ }_{2} \mathrm{~F}_{1}\left({ }_{2}^{2}, \frac{3}{2} \mid 2 J\right)\right)^{2}\right]^{\frac{1}{2}} .
$$

Then we obtain for a Mandel parameter $Q=\frac{(\Delta n)^{2}}{\langle n\rangle}-1$ a relation

$$
Q=\frac{{ }_{2} \mathrm{~F}_{1}\left(\frac{3}{2}, \frac{5}{2} \mid 2 J\right)}{{ }_{2} \mathrm{~F}_{1}\left(\begin{array}{c}
\frac{3}{2}, \frac{5}{2} \\
2
\end{array} \mid 2 J\right)}-\frac{3}{2} J \frac{{ }_{2} \mathrm{~F}_{1}\left(\begin{array}{c}
\frac{3}{2}, \frac{5}{2} \\
2
\end{array} \mid 2 J\right)}{{ }_{2} \mathrm{~F}_{1}\left(\frac{1}{2}, \frac{3}{2} \mid 2 J\right)}-1 .
$$

\section{Conclusion.}

In the present note we have defined a new type of an oscillator for which the Legendre polynomials play the same role as the Hermite polynomials play for standard boson oscillator.Solving the appropriate classical moment problem,we defined two sets of coherent states - as eigenvectors of an annihilation operator and temporary stable coherent state of the Klauder - Gazeau type. In our next work which is in the closing stage, we shall define the Perelomov type coherent states for the Legendre oscillator, as once more concrete example of general study of connections between orthogonal polynomials and coherent states. Similar oscillator-like systems can be defined for others orthogonal polynomials [1] (including $q$-deformed ones). For these systems one can also define the corresponding systems of coherent states (the rather general construction we will to describe in our following work). 


\section{References}

[1] V.V.Borzov, Orthogonal polynomials and generalized oscillator algebras, Integral Transf. and Special Funct. 12, no.2, 115-138 (2001)

[2] V.V.Borzov, E.V.Damaskinsky, P.P.Kulish, Construction of the spectral measure for the deformed oscillator position operator in the case of undetermined Hamburger moment problem, Reviews in Math. Phys., 12, no.5, 691-710 (2000).

[3] E.V.Damaskinsky and P.P.Kulish, Deformed oscillators and their applications, Zap. Nauch. Sem. LOMI, 189, 37-74, (1991) (in Russian), English transl: J. Soviet. Math. 62, 2963 (1992).

[4] V.V.Borzov, E.V.Damaskinsky, Realization of the annihilation operator for generalized oscillator-like system by a differential operator and Hermite-Chihara polynomials, Integral Transf. and Special Funct. 13(6) 547-554 (2002).

[5] A.O.Barut, L.Girardello, New "Coherent States" Associated with Non-Compact Groups, Commun. Math.Phys. 21, no.1, 41-55 (1972).

[6] J.P.Gazeau and J.R.Klauder, Coherent states for systems with discrete and continuous spectrum, J. Phys. A.: Math. \& Gen. 32, no.1, 123-132(1999).

[7] A.M.Perelomov, Coherent States for Arbitrary Lie Groups, Commun. Math. Phys. 26, 222-236 (1972).

[8] A.M.Perelemov, Generalized coherent states and some of their applications. Sov. Phys. Usp. 20, 703-720 (1977);

[9] A.M.Perelomov, Generalized Coherent States and Their Applications (Springer-Verlag, Berlin, 1986).

[10] N.I.Akhieser, The classical moment problem and some related questions in analysis, Hafner Publ.Co, New York, 1965.

[11] M.S.Birman and M.Z.Solomyak, Spectral theory of self-adjoint operators in Hilbert space, Leningrad Univ. Press, 1980 (in Russian).

[12] J-M.Sixdenierrs, K.A.Penson, A.I.Solomon, Mittag-Leffler coherent states, J. Phys. A. 32, no.43, 7543-7563(1999).

[13] I.S.Gradstein, I.M.Ryzhik, Table of integrals, series and products, Academic Press, 1980. 
[14] A.P.Prudnikov, Yu.A.Brychkov, O.I.Marichev: "Integrals and series. Elementary functions." (in Russian) Nauka, Moscow, 1981 799pp. 